\title{
In-Situ Melting Behavior Observation of an Eutectic Alloy Nano-Needle
}

\author{
Katsuhiro Sasaki ${ }^{1}$, Takuya Takahashi ${ }^{1}$, Shigeo Arai $^{2}$ Tomoharu Tokunaga $^{1}$ and Takahisa Yamamoto ${ }^{1}$ \\ 1. Department of Quantum Engineering, Nagoya University, Nagoya, 464-8603, Japan. \\ 2. EcoTopia Science Institution, Nagoya University, Nagoya, 464-8603, Japan.
}

Metal wedge [1] and nano-needle [2] shape specimen embedded in amorphous carbon film can sustain stable liquid phase at elevated temperatures in a vacuum of the transmission electron microscope (TEM). The behavior of the solid-liquid interface in wedge and needle shape of pure metal has been understood $[1,2]$ as a relationship among the interface energies of solid-solid and solid-liquid interfaces. In the eutectic alloy with larger latent heat at around eutectic point, the solid-liquid interface could show different behavior. An Au-Si eutectic alloy nano-needle has been observed by in-situ heating experiment in a TEM.

A (001) Si wafer was cleave along 110 surface and the surface oxide layer was removed with using a DC glow discharge (Fig. 1a) at $8 \mathrm{~Pa}$ of Ar-5at $\% \mathrm{H}_{2}$. After the surface cleaning, 400 $\mathrm{nm}$ of $\mathrm{Au}$ was deposited (Fig. 1b) on the surface by changing the polarity of the discharge without exposing the sample surface to the air. Diamond particles with $5 \mu \mathrm{m}$ of diameter were dispersed (Fig. 1c) on the surface of $\mathrm{Au}$ coated $\mathrm{Si}$ substrate. The nano-needles were formed using the ion shadow method [2] which irradiates the sample surface vertically (Fig. 1d and e) with $4.5 \mathrm{keV}$ of Ar ion beam. The surface of the prepared sample were covered by the $20 \mathrm{~nm}$ of plasma polymerized CVD film (Fig. 1f) formed by the DC discharge in $4 \mathrm{~Pa}$ of $\mathrm{CH}_{4}$ and $\mathrm{C}_{2} \mathrm{H}_{4}$. In-situ heating experiment was performed on an ordinary heating holder in a high voltage TEM JEOL 1000K RS at an accelerating voltage of $1 \mathrm{MV}$.

As shown in Fig. 2a, the nano-needle consisted of a diamond particle on top and $400 \mathrm{~nm}$ height of a Au truncated corn kept on a Si truncated corn with $1.5 \mu \mathrm{m}$ of height at room temperature. The Au runcated corn became molten at $600 \mathrm{~K}$ which was measured by the thermocouple attached to the heating holder furnace. Thermodynamical calculation suggests that depression of meting temperature with the size of the needle as shown in Fig. 2 should be less than few degree. The phase diagram of Au-Si system [4] indicates the eutectic temperature to be $636 \mathrm{~K}$. This difference could be deduced as the temperature difference between thermocouple and observed area. Namely, the measured temperature could be adjusted by adding $36 \mathrm{~K}$ of the difference. The $\mathrm{Au}-\mathrm{Si}$ liquid extended into $\mathrm{Si}$ increasing the length linearly as a function of temperature as shown in Fig $2 \mathrm{~b}$ to d. In pure metal specimen, the length of liquid showed inverse proportional behavior as a function of temperature [3]. The difference suggests that the amount of liquid in partially molten eutectic alloy needle will be determined by the phase diagram, which results linear increase of liquid length, instead of the relationship among solid-solid and solid-liquid interfaces. During heating up to $673 \mathrm{~K}$, the temperature and the amount of liquid does not affect the nano-needle shape. This suggests that the molten Au-Si liquid is tightly kept by the plasmapolymerized film in the vacuum of TEM. The position and the shape of the solid-liquid interface between the diamond particle and Au-Si liquid was unchanged during heating experiment. However, the solid-liquid interface between $\mathrm{Si}$ and Au-Si liquid changed hemispherical shape caved in Au-Si liquid as indicated with white dotted caves in Fig $2 \mathrm{~b}$ to $\mathrm{d}$. The contacting angle between the solid-liquid interface and the side surface of the needle are measured to be $43^{\circ}, 24^{\circ}, 19^{\circ}$ and $14^{\circ}$ at $603 \mathrm{~K}, 623 \mathrm{~K}$ (Fig.2b), 643K (Fig.2c) and 663K (Fig. 2d), respectively. These large change could correspond the change of the solid-liquid interface energy due to the compositional change of Au-Si liquid along the liquidus line 
shown in the phase diagram [4]. At $400 \mathrm{~K}$, minimum contacting angle shows $4^{\circ}$ which results expansion of few nm of thin liquid layer along the side surface of the needle. This thin layer of liquid could allow us to observe the solid-liquid interface in an atomic resolution.

We have observed melting behavior of Au-Si eutectic alloy nano-needle. The linear increase of liquid length suggests that the melting behavior obeys phase diagram instead of the relationship among the interfaces. The observation of eutectic reaction suggests a method of temperature adjustment of heating holder. We deduce that the stable liquid formation in a wide range of temperature could result a possibility of direct temperature measurement on a local position in a TEM specimen. Also, the formation of few $\mathrm{nm}$ of stable liquid layer could allow us to observe the solid-liquid interface in an atomic resolution.

References:

[1] Y. Senda, K. Sasaki and H. Saka, Philos. Mag. 84, (2004), pp. 2635-2649.

[2] J. Chang, E. Johnson, T Sakai, and H. Saka, Philos. Mag., 89, (2009), pp. 595-604.

[3] K. Sasaki, M. Hattori, S. Arai, N. Tanaka, K. Kuroda, T. Yamamoto, Conference Proceedings APMC 10 / ICONN 2012 / ACMM 22, (2012), pp. 989-1,-2

[4] "Binary alloy phase diagrams," ed. T. B. Massalski, (American Society of Metals, Metals Park, Ohio, USA) 1986.
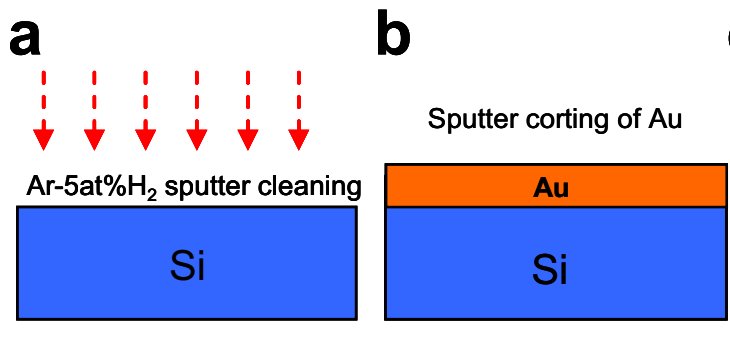

C Few micrometer size particles with low thinning rate (Diamond)
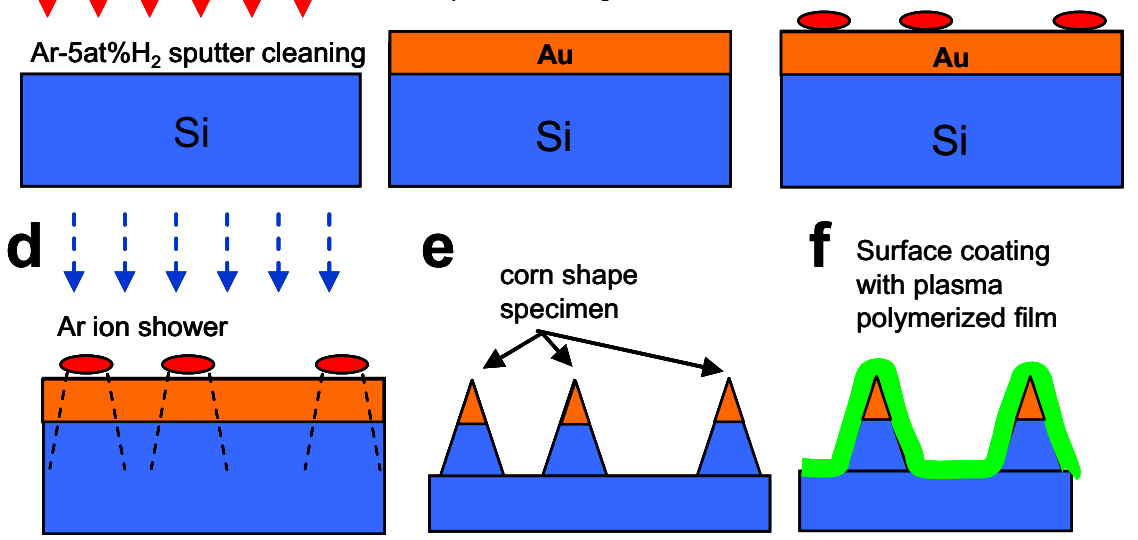

Surface coating
with plasma polymerized film
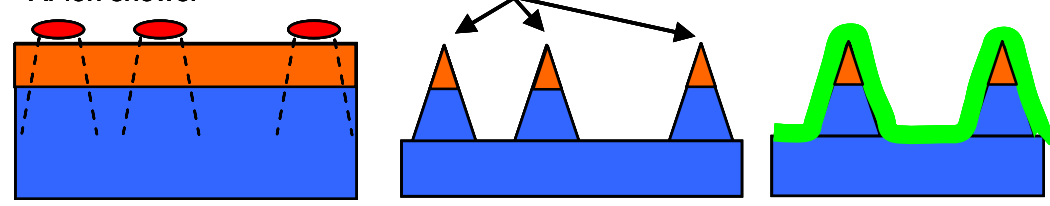

Figure 1. Preparation procedure of Au-Si nano-needle

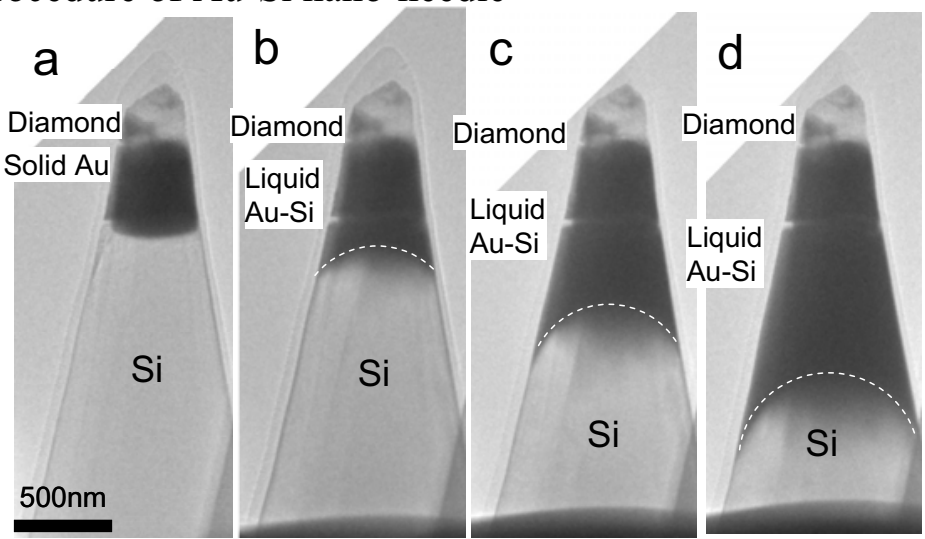

Figure 2. Partial melting process of an Au-S nano-needle at (a)RT, (b) $623 \mathrm{~K}$, (c) $643 \mathrm{~K}$ and (d) $663 \mathrm{~K}$. 